\title{
Early Laparoscopic Resection of Ruptured Hepatocellular Carcinoma
}

\author{
Brian K. P. Goh, MBBS, MMed, MSc, FRCS, Lip-Seng Lee, MBBS, MRCS, \\ Pierce K. H. Chow, MBBS, MMed, PhD, FRCS \\ Department of Surgery, Singapore General Hospital, Singapore, Singapore (Drs. Goh, Lee, Chow). \\ Duke-NUS Graduate Medical School, Singapore, Singapore (Drs. Goh, Chow).
}

\begin{abstract}
Introduction: Laparoscopic liver resection has increasingly been used as a therapeutic modality in hepatocellular carcinoma (HCC). However, its use in the treatment of spontaneously ruptured HCC has not been well documented.

Case Description: We describe a case of spontaneously ruptured HCC successfully treated with early totally laparoscopic liver resection.

Discussion: Early laparoscopic resection of ruptured HCC is feasible and should be considered in the treatment algorithm of selected patients who have been well stabilized.
\end{abstract}

Key Words: Laparoscopy, Hepatectomy, Hepatocellular carcinoma, Ruptured, HCC, Resection.

\footnotetext{
Citation Goh BKP, Lee L-S, Chow PKH. Early laparoscopic resection of ruptured hepatocellular carcinoma. CRSLS e2013.00010. DOI: 10.4293/CRSLS.2013.00010.

Copyright (C) 2015 by SLS, Society of Laparoendoscopic Surgeons. This is an open-access article distributed under the terms of the Creative Commons Attribution-Noncommercial-ShareAlike 3.0 Unported license, which permits unrestricted noncommercial use, distribution, and reproduction in any medium, provided the original author and source are credited.

All authors do not have any conflict of interest financially or otherwise and have nothing to disclose.

Address correspondence to: Brian K. P. Goh, MBBS, MMed, MSc, FRCS, Hepatopancreatobiliary and Transplantation Service, Department of Surgery, Singapore General Hospital, Outram Road, Singapore 169608, Singapore. E-mail: bsgkp@hotmail.com. Phone: +65-63214051
}

\section{INTRODUCTION}

Laparoscopic liver resection was first reported by Gagner et al in 1992. ${ }^{1}$ However, because of its complexity, laparoscopic liver was not widely adopted and the first large case series was only reported 8 years later, in 2000 , by Cherqui et $\mathrm{al}^{2}$ in 30 patients. Since then, with the introduction of various new and improved laparoscopic devices, laparoscopic liver resection has been increasingly adopted worldwide and numerous series have been published in the literature. ${ }^{3-5}$ Laparoscopic liver resection has been shown to be superior to open liver resection in terms of postoperative outcomes such as decreased pain and shorter hospital stay. ${ }^{3-5}$ It has also been shown to be safe with blood loss, blood transfusion rates, postoperative morbidity rates, and postoperative mortality rates that are similar to, if not lower than, those with open surgery. ${ }^{3-6}$ Numerous investigators have also confirmed its effectiveness for treating malignant lesions such as hepatocellular carcinoma (HCC), reporting similar tumor-free margins and survival rates compared with open resection. ${ }^{6-8}$
Spontaneously ruptured HCC has been reported to occur in $3 \%$ to $15 \%$ of HCC patients and is associated with a high mortality rate of $25 \%$ to $75 \%$ due to liver failure. ${ }^{1,9}$ The initial management of ruptured HCC involves early resuscitation, stabilization, and hemostasis. In most instances, a staged liver resection is advocated as the preferred definitive treatment. ${ }^{1,9}$ This is because 1 -stage emergency liver resection has been reported to have a dismal resection rate of only $12.5 \%$ to $31 \%$ and mortality rate of $16.5 \%$ to $100 \% .1,9$ However, 1-stage emergency liver resection can still be considered in stabilized patients with an easily accessible tumor and good functional liver reserve.?

In this study we report a case of spontaneously ruptured HCC treated by early laparoscopic resection. To our knowledge, this is the first study to describe early laparoscopic resection for spontaneously ruptured HCC. Only 1 study has previously described and discussed the role of delayed laparoscopic resection in the treatment of ruptured liver tumors. ${ }^{1}$ 


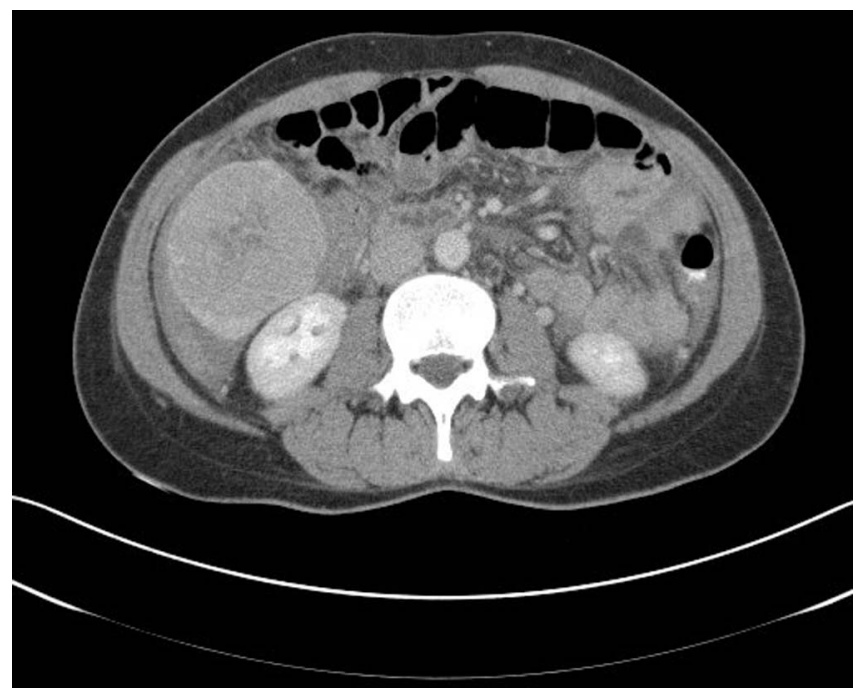

Figure 1. Computed tomography scan of 6-cm ruptured HCC with surrounding hemoperitoneum.

\section{CASE REPORT}

A 51-year-old female known hepatitis B carrier was admitted to the emergency department with generalized abdominal pain of 2 days' duration that was progressively worsening. On physical examination, she was hypotensive with a systolic blood pressure of 77 $\mathrm{mm} \mathrm{Hg}$ and tenderness mainly over her right hypochondrial and flank region. Her hemoglobin level was $10.7 \mathrm{~g} / \mathrm{dL}$. The rest of her serum biochemistry panel including liver function tests was unremarkable. She was resuscitated with fluid and remained hemodynamically stable in the high dependency unit. Computed tomography scan performed the next day showed a 6.0-cm exophytic mass arising from segment V/VI with surrounding hemoperitoneum and hematoma but no active hemorrhage (Figures $\mathbf{1}$ and 2 ). The serum $\alpha$-fetoprotein level was elevated at $303 \mu \mathrm{g} / \mathrm{L}$. The patient was diagnosed with likely ruptured HCC on the background of Child A cirrhosis. A repeat hemoglobin level was obtained and was $7.8 \mathrm{~g} / \mathrm{dL}$, and she was transfused with $2 \mathrm{U}$ of blood. The patient underwent emergency laparoscopic nonanatomic resection of the ruptured HCC approximately 26 hours after her presentation to the hospital. The procedure was performed by use of a 12-mm umbilical port for the laparoscope (A), two $5-\mathrm{mm}$ and $12-\mathrm{mm}$ epigastric ports (B and $\mathrm{C}$ ), and a 5-mm right iliac fossa port (D) (Figure 3). Laparoscopy confirmed the ruptured liver tumor with $1 \mathrm{~L}$ of blood in the peritoneal cavity. The tumor margins were confirmed by intraoperative ultrasonography and resection

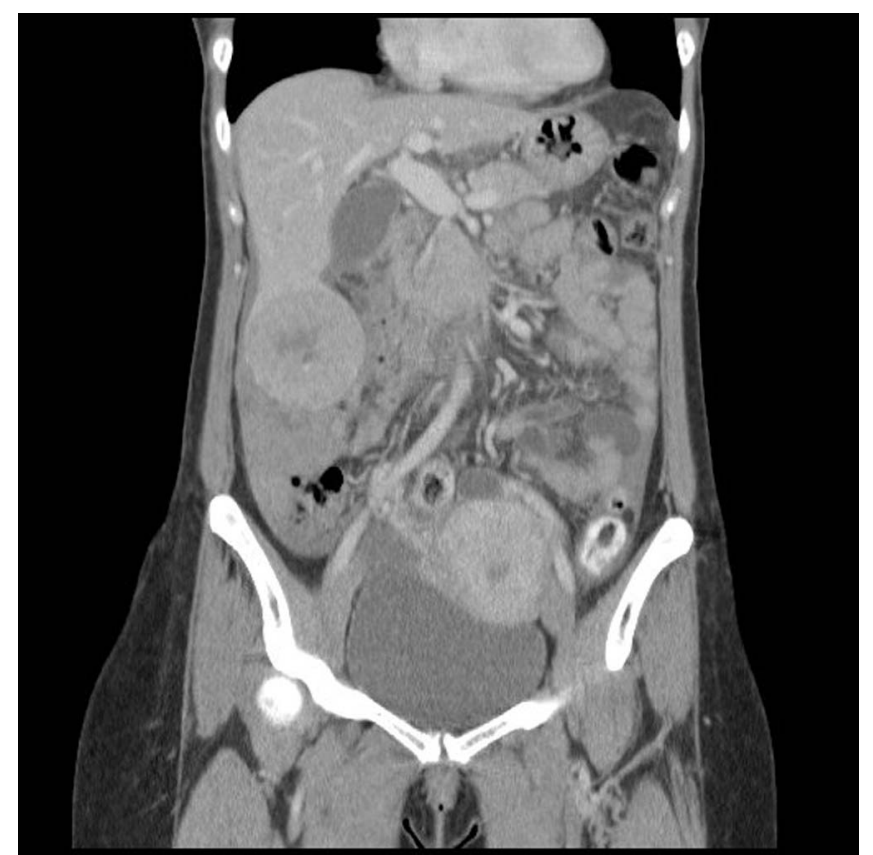

Figure 2. Computed tomography scan (coronal view) of the ruptured HCC.

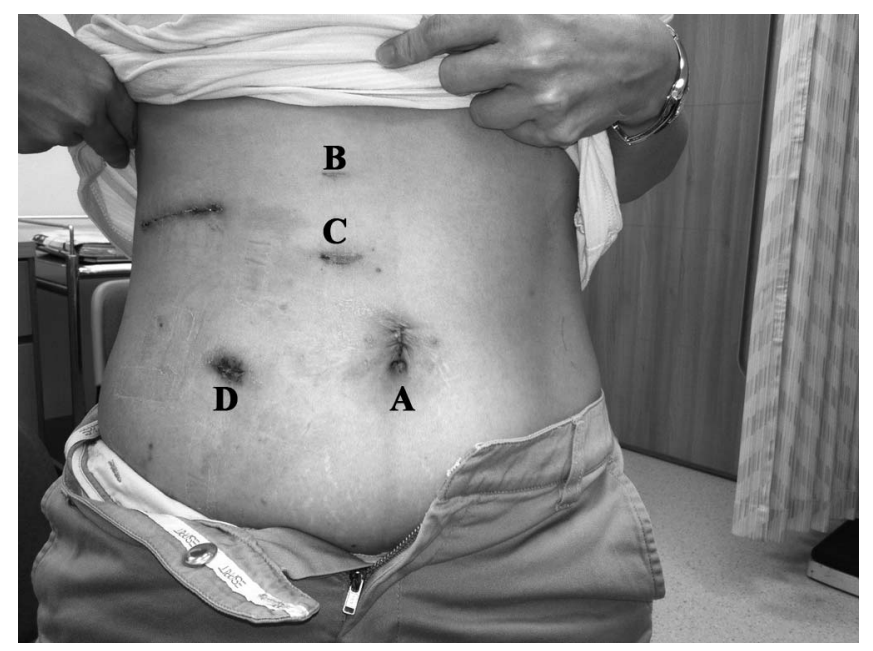

Figure 3. Port placements and incisions on patient's abdomen.

margins marked by cautery. The liver and tumor were not mobilized to minimize manipulation and prevent further potential spillage of tumor cells. Parenchymal transection was performed with Ligasure (Covidien, Boulder, CO, USA) without application of the Pringle maneuver. An endovascular stapler was used to transect the main vascular pedicle. A $6-\mathrm{cm}$ incision over the right hypochondrium was made to extract the specimen through a specimen bag. After completion of the resec- 
tion, a thorough washout of the entire abdominal cavity was performed with $3 \mathrm{~L}$ of fluid. A right hypochondrial incision was used to extract the specimen instead of the less painful periumbilical incision or Pfannenstiel incision because this facilitated thorough washout of the surgical site through the open incision and minimized the potential for further tumor spillage compared with tumor extraction through a distant site. The operation time was 170 minutes, and the estimated blood loss was $300 \mathrm{~mL}$. The patient's postoperative recovery was uneventful, and she was discharged on postoperative day 7. Histology confirmed a $6-\mathrm{cm}$ ruptured HCC with a 2 -cm tumor-free resection margin.

\section{DISCUSSION}

HCC is the fifth most common cancer worldwide, and spontaneous rupture has been reported to occur in up to $15 \%$ of patients. 9 Ruptured HCC is associated with a high mortality rate of $25 \%$ to $75 \% .{ }^{9}$ The most important prognostic factors include the stage of the cancer, functional liver reserve, and degree of shock at presentation. The optimal treatment of ruptured HCC has not been well defined, although the initial management almost always includes adequate resuscitation with hemostasis. Presently, the most commonly adopted and advocated approach would be initial stabilization and hemostasis through transarterial embolization followed by staged hepatic resection..$^{9,10}$ In patients who are stable with no clinical or radiologic evidence of continuous bleeding, conservative management without a hemostatic procedure may be advocated. ${ }^{11}$ In this day, open surgical hemostasis is reserved as a second-line treatment in the event of failed transarterial embolization. ${ }^{9}$

Liver resection is the only curative option for ruptured HCC. However, there is controversy regarding the optimal timing for resection either as an emergency singlestage liver resection or as a 2-stage hepatic resection. Proponents of single-stage surgery argue that a delay in resection after hemostasis may compromise the resection rate because of tumor dissemination. 9 However, given the high inpatient mortality rates of $16.5 \%$ to $100 \%$ associated with emergency resection, most authors today advocate staged liver resection, which is associated with inpatient mortality rates of $0 \%$ to $9 \% .9$ The poor outcome associated with emergency surgery is likely related to the unclear liver functional reserve and the high surgical risk in patients with hypovolemic shock. ${ }^{9}$ Nonetheless, early 1 -stage surgery may still be considered in highly selected patients who are hemodynamically stable with no ongoing bleeding and good functional liver reserve.

Laparoscopic liver resection is now well accepted in the treatment of HCC because it offers the usual benefits of minimally invasive surgery and has been shown to have oncologic outcomes similar to open resection. ${ }^{4,6-8}$ However, its use in the treatment of ruptured HCC has not been well documented. In general, the use of laparoscopy in the setting of hemoperitoneum and shock has been limited because of concerns about visibility, exposure, and prolonged operating times. ${ }^{1,12}$ Only 1 previous study has addressed the role of laparoscopic liver resection in the treatment of ruptured liver tumors. ${ }^{1}$ In the study by Belgaumkar et al, ${ }^{1} 3$ patients, 2 with ruptured HCC and 1 with ruptured hepatic adenoma, underwent successful laparoscopic liver resection. The 3 patients underwent surgery 4 days, 12 days, and 21 days after presentation. To our knowledge, the current report is the first to describe early laparoscopic liver resection as a treatment modality for ruptured HCC. This report shows that in a highly selected patient who can be hemodynamically stabilized and has no evidence of continuous active bleeding, good liver function, and easily accessible tumor, early laparoscopic liver resection can be considered a therapeutic option.

In conclusion, early laparoscopic resection of ruptured HCC is feasible and should be considered in the treatment algorithm of selected patients who have been well stabilized.

\section{References:}

1. Belgaumkar A, Carswell KA, Patel AG. Laparoscopic resection of ruptured tumors. J Laparoendosc Adv Surg Tech A. 2009; 19(5):641-645.

2. Cherqui D, Laurent A, Tayar C, Chang S, et al. Laparoscopic liver resection for peripheral hepatocellular carcinoma in patients with chronic liver disease: midterm results and perspectives. Ann Surg. 2006;243:499-506.

3. Buell JF, Cherqui D, Geller DA, O'Rourke N, et al. The international position on laparoscopic liver surgery: the Louisville statement, 2008. Ann Surg. 2009;250:825-830.

4. Nguyen KT, Gamblin TC, Geller DA. World review of laparoscopic liver resection-2,804 patients. Ann Surg. 2009;250:831841. 
5. Laurence JN, Lam VW, Langcake ME, Hollands MJ, Crawford MD, Pleass HC. Laparoscopic hepatectomy, a systematic review. ANZ J Surg. 2007;77:948-953.

6. Fancellu A, Rosman AS, Sanna V, et al. Meta-analysis of trials comparing minimally-invasive and open liver resections for hepatocellular carcinoma. J Surg Res. 2011;171(1):e33e45.

7. Chen HY, Juan CC, Ker CG. Laparoscopic liver surgery for patients with hepatocellular carcinoma. Ann Surg Oncol. 2008; 15:800-806.

8. Belli G, Fantini C, D'Agostino A, et al. Laparoscopic versus open liver resection for hepatocellular carcinoma in patients with histologically proven cirrhosis: short- and middle-term results. Surg Endosc. 2007;21:2004-2011.
9. Lai EC, Lau WY. Spontaneous rupture of hepatocellular carcinoma: a systematic review. Arch Surg. 2006;141:191-198.

10. Kung CT, Liu BM, Ng SH, et al. Transcatheter arterial embolization in the emergency department for hemodynamic instability due to ruptured hepatocellular carcinoma: analysis of 167 cases. AJR Am J Roentgenol. 2008;191:W231-W239.

11. Leung KL, Lau WY, Lai PB, Yiu RY, Meng WC, Leow CK. Spontaneous rupture of hepatocellular carcinoma. Arch Surg. 1999;134:1103-1107.

12. Sauerland S, Agresta F, Bergamashi R, et al. Laparoscopy for abdominal emergencies: evidence-based guidelines of the European Association for Endoscopic Surgery. Surg Endosc. 2006;20: $14-29$. 